\title{
Estimating the impact of a novel drug regimen for treatment of tuberculosis: a modeling analysis of projected patient outcomes and epidemiological considerations
}

\author{
Emily A. Kendall ${ }^{1 *}$ (D), Shelly Malhotra ${ }^{2,3}$, Sarah Cook-Scalise ${ }^{2}$, Claudia M. Denkinger ${ }^{4,5}$ and David W. Dowdy
}

\begin{abstract}
Background: Regimens that could treat both rifampin-resistant (RR) and rifampin-susceptible tuberculosis (TB) while shortening the treatment duration have reached late-stage clinical trials. Decisions about whether and how to implement such regimens will require an understanding of their likely clinical impact and how this impact depends on local epidemiology and implementation strategy.

Methods: A Markov state-transition model of 100,000 representative South African adults with TB was used to simulate implementation of the regimen BPaMZ (bedaquiline, pretomanid, moxifloxacin, and pyrazinamide), either for RR-TB only or universally for all patients. Patient outcomes, including cure rates, time with active TB, and time on treatment, were compared to outcomes under current care. Sensitivity analyses varied the drug-resistance epidemiology, rifampin susceptibility testing practices, and regimen efficacy.

Results: Using BPaMZ exclusively for RR-TB increased the proportion of all RR-TB that was cured by initial treatment from $60 \pm 1 \%$ to $67 \pm 1 \%$. Expanding use of BPaMZ to all patients increased cure of RR-TB to $89 \pm 1 \%$ and cure of all TB from $87.3 \pm 0.1 \%$ to $89.5 \pm 0.1 \%$, while shortening treatment by 1.9 months/person. In sensitivity analyses, reducing the coverage of rifampin susceptibility testing resulted in lower projected proportions of patients cured under all regimen scenarios (current care, RR-only BPaMZ, and universal BPaMZ), compared to the proportions projected using South Africa's high coverage; however, this reduced coverage resulted in greater expected incremental benefits of universal BPaMZ implementation, both when compared to RR-only BPaMZ implementation and when compared to to current care under the same low rifampin susceptibility testing coverage. In settings with higher RR-TB prevalence, the benefits of BPaMZ were magnified both for RR-specific and universal BPaMZ implementation.
\end{abstract}

Conclusions: Novel regimens such as BPaMZ could improve RR-TB outcomes and shorten treatment for all patients, particularly with universal use. Decision-makers weighing early options for implementing such regimens at scale will want to consider the expected impact on patient outcomes and on the burden of treatment in their local context.

Keywords: Tuberculosis, Treatment, Regimen selection, Drug resistance, Novel regimens, Clinical outcomes, Modeling

\footnotetext{
* Correspondence: ekendall@jhmi.edu

${ }^{1}$ Division of Infectious Diseases and Center for Tuberculosis Research, Johns

Hopkins University School of Medicine, CRB2 Room 106, 1550 Orleans St,

Baltimore, MD 21287, USA

Full list of author information is available at the end of the article
}

(c) The Author(s). 2019 Open Access This article is distributed under the terms of the Creative Commons Attribution 4.0 International License (http://creativecommons.org/licenses/by/4.0/), which permits unrestricted use, distribution, and reproduction in any medium, provided you give appropriate credit to the original author(s) and the source, provide a link to the Creative Commons license, and indicate if changes were made. The Creative Commons Public Domain Dedication waiver (http://creativecommons.org/publicdomain/zero/1.0/) applies to the data made available in this article, unless otherwise stated. 


\section{Background}

Annually, ten million people develop tuberculosis (TB), and more than one million die of TB [1]. Treatment remains arduous, and relapse rates after first-line treatment exceed 5\% [2]. For TB that is rifampin-resistant (RR) or multidrug-resistant, the necessities of an additional drug-resistant detection step and of treatment lasting 9 to 18 months pose even greater challenges. Shorter and more universally effective regimens which treat both rifampin-susceptible (RS) and RR-TB in 6 months or less could be transformative [3].

One potential such regimen in late-stage clinical development is BPaMZ, which combines the novel TB drugs bedaquiline $(\mathrm{B})$ and pretomanid $(\mathrm{Pa})$ with the first-line TB drug pyrazinamide $(\mathrm{Z})$ and the third-generation fluoroquinolone moxifloxacin (M). Data from the $\mathrm{NC}$ 005 Phase 2B human study suggest that at 8 weeks, even subsets of the BPaMZ regimen - namely, BPaZ for drug-susceptible $\mathrm{TB}$, or $\mathrm{BPaMZ}$ in patients with pyrazinamide-resistant strains of multidrug-resistant TB - surpassed the performance of the current standard regimen (HRZE [isoniazid, rifampin, pyrazinamide, and ethambutol]) in pan-susceptible TB [4]. In murine models, BPaMZ demonstrated powerful sterilizing activity as well, and even $\mathrm{BPa}$ alone rivaled the efficacy of HRZE [5]. A phase 2c/3 trial of the BPaMZ regimen (SimpliciTB) is underway, evaluating its potential to both shorten treatment to 4 months for patients with drugsusceptible TB and also effectively treat (as a 6-month regimen, because of their higher risk of associated resistance to pyrazinamide) patients with rifampin- or multidrug-resistant TB [6].

If this regimen continues to prove successful, policy-makers will need to understand its expected impact on clinical outcomes in order to implement it effectively and allocate resources appropriately. A key decision is whether to adopt the regimen for patients with rifampin-resistant $\mathrm{TB}$, rifampin-susceptible, or both, and to what extent this decision depends upon local epidemiology.

We therefore constructed a model of clinical outcomes among a hypothetical cohort of people with TB in South Africa. Our primary objective was to quantify the potential epidemiological benefit and adverse consequences (e.g., emergence of drug resistance) associated with this regimen.

\section{Methods}

\section{Simulated cohort and Markov model}

We developed a Markov model to simulate the course of TB disease for a cohort of people with pulmonary TB (Fig. 1). Using this model, we followed individuals under different algorithms for BPaMZ regimen use and different underlying epidemiologic and regimen-efficacy assumptions, considering outcomes of cure, time with $\mathrm{TB}$, prevalence and acquisition of drug resistance, and drug utilization.

Individuals were characterized according to TB status, time since TB onset, previous TB treatment, HIV infection status, smear status at time of diagnosis, and susceptibility to each of six different drug classes. The initial characteristics within the cohort reflected their distribution and correlations among notified TB in South Africa, from data sources including notifications to the national program [1] and drug resistance surveys [7, 8] (Table 1, Additional file 1: Table S1).

Cases were followed from the onset of a new or recurrent TB episode. Modeled events included TB diagnosis, Xpert-based rifampin DST where available, regimen selection, treatment (modeling duration as the number of months prescribed, or fewer if loss to follow up occurred), and treatment outcomes of either cure, or failure or relapse (with or without newly acquired drug resistance). Diagnosis and treatment could occur up to four times if initial diagnosis did not lead to curative treatment. Parameters are listed in Table 1 and in Additional file 1: Table S2.

\section{Projecting individual treatment outcomes}

The probabilities of achieving cure, and of acquiring drug resistance if not cured, each depended on the combined effects of all prescribed drugs to which the patient's disease was susceptible. Probability of cure additionally depended on the duration of treatment completed.

In the absence of data on clinical cure after BPaMZ, probabilities of cure were extrapolated from probabilities of culture conversion at 8 weeks in clinical trials $[4,20]$, using a regression model derived from historical trial data [21]. We additionally imposed the assumption that for drug-susceptible TB, 4 months of BPaMZ had a relapse rate equivalent to 6 months of HRZE - consistent with culture conversion data and current trial design [4, 6]. Details are provided in Additional file 1, including Tables S3 and S4, with select cure probabilities summarized in Table 2.

Although BPaMZ does not contain rifampin, the currently proposed strategy for assignment of BPaMZ treatment duration leverages the known association between resistance to rifampin (for which accurate, rapid DST is increasingly performed) and resistance to pyrazinamide as a component of BPaMZ. [6, 7]. Accordingly, we assumed that BPaMZ would be prescribed for 6 months for patients known to have RR-TB, and for 4 months otherwise. This is a pragmatic strategy that acknowledges that fluoroquinolone resistance testing is not performed widely. 


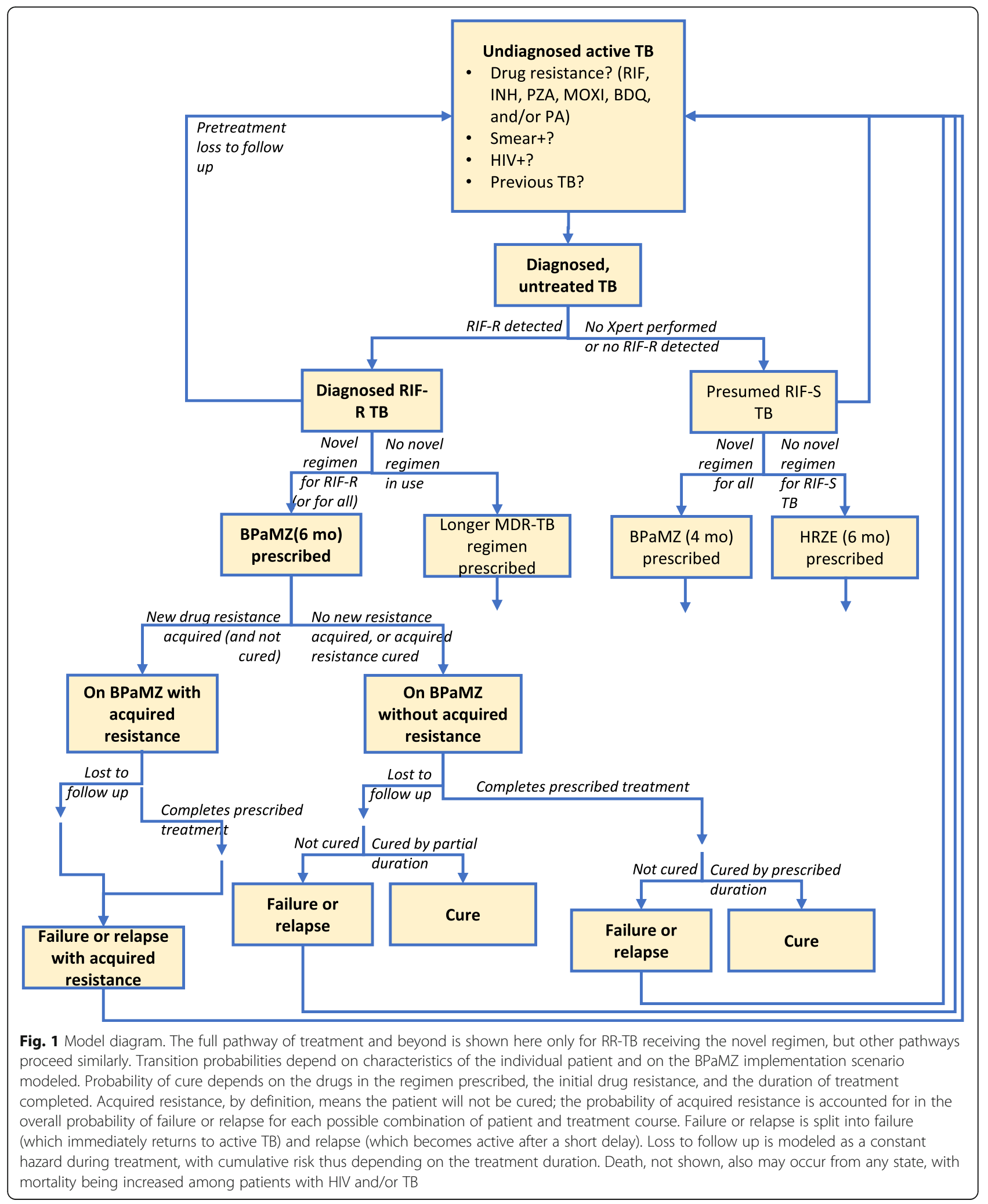

For parameters describing the risk of acquiring drug resistance during treatment, our primary analysis assumed that when pan-susceptible TB was treated with
$\mathrm{BPaMZ}$, the combined risk of acquiring resistance to any of the included drugs (except for pyrazinamide) equaled the risk of acquiring resistance to rifampin when treated 
Table 1 Select model parameters

\begin{tabular}{|c|c|c|c|}
\hline Parameter & Estimate, South Africa & Range in sensitivity analysis & References and notes \\
\hline Fraction of TB cases previously treated for TB & $10 \%$ & $8-13 \%$ & [9] \\
\hline Fraction of TB cases with HIV & $60 \%$ & $54-66 \%$ & [1] \\
\hline Baseline prevalence of RR, new cases & $3.4 \%$ & $2.5-4.3 \%$ & [1] \\
\hline Baseline prevalence of RR, cases previously treated for TB & $7.1 \%$ & $4.8-9.5 \%$ & [1] \\
\hline Prevalence of pyrazinamide resistance, if RR & $44 \%$ & $33-55 \%$ & [7] \\
\hline Prevalence of pyrazinamide resistance, if RS & $1.3 \%$ & $0.8-2.0 \%$ & [7] \\
\hline Prevalence of any moxifloxacin resistance, if RR & $9.5 \%$ & $4-18 \%$ & {$[7,10]$} \\
\hline Prevalence of high-level moxifloxacin resistance, if RR & $5.9 \%$ & $2-12 \%$ & {$[7,10]$} \\
\hline Prevalence of any moxifloxacin resistance, if RS & $0.4 \%$ & $0-0.9 \%$ & {$[7,10]$} \\
\hline Mean time from TB onset to TB diagnosis & 9 months & $6-15$ & $\begin{array}{l}\text { Incidence:prevalence ratio } \\
\text { estimates [1] }\end{array}$ \\
\hline Pretreatment loss to follow up & $10 \%$ & $5-20 \%$ & [11] \\
\hline Monthly loss to follow up during treatment & $1 \%$ & $0.8-2 \%$ & [12] \\
\hline Monthly TB mortality, untreated active TB & $2.1 \%$ & $2.3-2.8 \%$ & {$[1,13]$} \\
\hline Present-day Xpert MTB/RIF coverage, new patients & $70 \%$ & $60-80 \%$ & $\begin{array}{l}\text { [1] with projected increase to } \\
2019\end{array}$ \\
\hline $\begin{array}{l}\text { Present-day Xpert MTB/RIF coverage, patients previously } \\
\text { treated for TB }\end{array}$ & $75 \%$ & $60-90 \%$ & $\begin{array}{l}\text { [1] with projected increase to } \\
2019\end{array}$ \\
\hline $\begin{array}{l}\text { Relapse after six months HRZE or four months of BPaMZ } \\
\text { (assuming drug susceptibility) }\end{array}$ & $6.3 \%$ & $2-12 \%$ & {$[4,14-16]$; See Additional File 1.} \\
\hline $\begin{array}{l}\text { Odds ratio of cure from moxifloxacin-containing regimen, } \\
\text { low-level versus high-level moxifloxacin resistance }\end{array}$ & 1.7 & $1.3-2.2$ & $\begin{array}{l}\text { [17], based on levofloxacin vs } \\
\text { ofloxacin when ofloxacin resistant }\end{array}$ \\
\hline Risk of acquired RR after HRZE ${ }^{b}$ & 0.005 & $0.002-0.15$ & [18] \\
\hline Risks of acquired $\mathrm{B}, \mathrm{Pa}$, or $\mathrm{M}$ after $\mathrm{BPaMZ}{ }^{\mathrm{b}}$ & 0.002 & $0-0.01$ & Assumed \\
\hline $\begin{array}{l}\text { Risk of acquired moxifloxacin resistance after conventional } \\
\text { multidrug-resistant TB regimen }\end{array}$ & 0.04 & $0.005-0.08$ & [19] \\
\hline
\end{tabular}

$\mathrm{TB}=$ tuberculosis, $\mathrm{RS}=$ rifampin susceptible, $\mathrm{RR}=$ rifampin resistant, $\mathrm{Z}=$ pyrazinamide, $\mathrm{M}=$ moxifloxacin. $\mathrm{B}=$ bedaquiline, $\mathrm{Pa}=$ pretomanid, $\mathrm{HRZE}=$ standard first-line regimen of isoniazid, rifampin, pyrazinamide, ethambutol.

a The probability of successful treatment is reduced when resistance is present to one or more drugs in the regimen prescribed, or when duration is changed (shortened due to loss to follow up, or extended to six months for patients with RR-TB receiving BPaMZ), as shown in part $b$ of the Table

${ }^{b}$ Parameter value shown is the risk if initially susceptible to $R$ and $Z$ (HRZE), or to $B, P a$, and $M$ (BPaMZ). Risk of acquired resistance to remaining drugs is increased for $M$. tuberculosis strains already resistant to one or more of these drugs in the regimen used; see Additional File 1 for details

with HRZE. Remaining agnostic to which drug resistance would develop first, we divided the risk of acquired resistance equally between moxifloxacin, bedaquiline, and pretomanid. Pre-existing resistance increased the risk of acquiring resistance to additional drugs (Additional file 1: Tables S5 and S6).

\section{Implementation scenarios}

We considered the impact of BPaMZ in South Africa if all patients with $\mathrm{TB}$ were eligible for the new regimen ("Universal BPaMZ"), or if only those with known RRTB were eligible ("RR-only BPaMZ"), comparing each to outcomes under "Current Care".

In scenario analyses extrapolating settings other than South Africa, we also considered how projected impact changed with:
- Lower Xpert coverage (rifampin DST for only $10 \%$ of new patients and $37.5 \%$ [half the current level in South Africa] of retreatment patients), and/or

- Higher RR prevalence (3x higher odds of RR).

- Higher prevalence of moxifloxacin and pyrazinamide resistance (3-fold higher odds of each)

In sensitivity analyses, we evaluated how projections of BPaMZ impact were affected by:

- Lower BPaMZ efficacy (requiring 5 months of BPaMZ, rather than four, to achieve the efficacy of 6 months of HRZE)

- An improved multidrug-resistant TB standard of care (a 12-month regimen achieving outcomes similar to HRZE), reflecting ongoing improvements 
Table 2 Selected probabilities of durable cure, by active drugs and duration of treatment ${ }^{a}$

\begin{tabular}{|c|c|c|c|}
\hline Active drugs in prescribed regimen & 4 months & 6 months & 18 months \\
\hline$\overline{H R(Z E)}{ }^{b}$ & $86.0 \%^{c}$ & $94.4 \%$ & Not applicable \\
\hline$R(Z E)^{b}$ & $58.0 \%{ }^{\mathrm{c}}$ & $83.3 \%$ & Not applicable \\
\hline BPaMZ & $93.7 \%$ & $97.6 \%$ & Not applicable \\
\hline BPamZ $^{d}$ & $91.6 \%$ & $96.8 \%$ & Not applicable \\
\hline BPaM & $89.5 \%$ & $95.9 \%$ & Not applicable \\
\hline $\mathrm{BPaZ}$ & $86.5 \%$ & $94.6 \%$ & Not applicable \\
\hline BPam ${ }^{d}$ & $70.2 \%$ & $89.4 \%$ & Not applicable \\
\hline Conventional multidrug-resistant TB regimen with full fluoroquinolone activity $[22,23]$ & $20.0 \%^{c}$ & $40.2 \%^{\mathrm{c}}$ & $91.3 \%$ \\
\hline Conventional multidrug-resistant TB regimen in presence of fluoroquinolone resistance $[22,23]$ & $20.0 \%^{\mathrm{c}}$ & $20.0 \%{ }^{\mathrm{c}}$ & $79.1 \%$ \\
\hline
\end{tabular}

${ }^{a}$ Modeled as a function of two-month culture conversion and time on treatment, for the set of drugs in the prescribed regimen to which the patient's TB strain is susceptible. Details in Additional file 1

${ }^{b}$ Outcomes of HRZE are affected explicitly by isoniazid and/or rifampin resistance, but because data for the HRZE regimen come from studies that did not test for pyrazinamide or ethambutol resistance, outcomes are weighted averages reflecting the distribution of pyrazinamide and ethambutol resistance within each patient subpopulation

${ }^{c}$ Durations of 4 months for HRZE, and of 4 or 6 month for conventional MDR regimens, are shown for comparison but are not prescribed and are used within the model only if patients are lost to follow up at these time points

$\mathrm{d}$ " $\mathrm{m}$ " represents a moxifloxacin-containing regimen used to treat a TB strain that has low-level moxifloxacin resistance

in the efficacy, outcomes, and duration of multidrug-resistant TB treatment $[24,25]$

For outcomes specific to potential acquisition or transmission of drug-resistant TB, we also evaluated the effects of:

- Higher risks of BPaMZ resistance acquisition (including minimum 1\% risk of acquired resistance to each of moxifloxacin, bedaquiline, and pretomanid), or

- A nonzero (2\%) initial prevalence of bedaquiline resistance in the cohort, for example reflecting spontaneously-occurring resistance or clofazimine cross-resistance [26, 27].

Additional sensitivity analysis details are provided in Additional File 1.

\section{Reporting of outcomes}

We first repeatedly simulated disease courses for each possible set of baseline individual-patient characteristics (minimum 5000 times each; 50,000 times each for patient types comprising more than $5 \%$ of the cohort). From those simulated courses, we randomly sampled with replacement, weighting by the expected frequency of each set of patient characteristics within a South African cohort. We report results as a mean \pm standard deviation across 50 such cohorts of 100,000 people with TB.

Rather than a single combined utility, we measure and report the impact of regimen selection on multiple outcomes, including cure, time with active $\mathrm{TB}$ or active drug-resistant $\mathrm{TB}$, and months of $\mathrm{TB}$ treatment administered. TB cure, in particular, is also evaluated in several ways: We first consider the proportion of all individuals with TB who were cured within one diagnosis and treatment attempt, taking into account death before treatment, or initial loss to follow up, in the denominator (henceforth named "all individuals with TB"). We also consider the proportion cured among those individuals who were treated for TB (those who initiated a treatment regimen, independent of whether the regimen selected was appropriate for their TB strain or whether they completed the full regimen; henceforth named "patients treated"). Furthermore, we consider the proportion achieving within a certain number of months of TB onset, and the proportion ultimately achieving cure after multiple rounds of diagnosis and treatment.

\section{Results}

\section{BPaMZ and cure of RS- and RR-TB in South Africa}

The impact of the $\mathrm{BPaMZ}$ regimen among all individuals with TB, and among those all individuals with RR-TB, is shown in Fig. 2 (additional outcomes in Additional file 1: Table S7). Under Current Care, a single round of treatment cured only $45.0 \pm 0.7 \%$ of all individuals with RR-TB (including those never diagnosed or treated, and those whose resistance was not diagnosed), for reasons that include TB under-diagnosis, mortality before and after TB diagnosis, loss to follow up before and during treatment, failure and relapse after RR-TB treatment, and failure and relapse after HRZE treatment of undiagnosed RR-TB. BPaMZ use for only RR-TB increased this proportion cured to $50.3 \pm 0.8 \%$ of all individuals with RR-TB. Limiting analysis to RR-TB patients treated (i.e., to individuals with RR-TB who initiated any TB treatment), the proportion cured was $60.0 \pm 0.9 \%$ under 


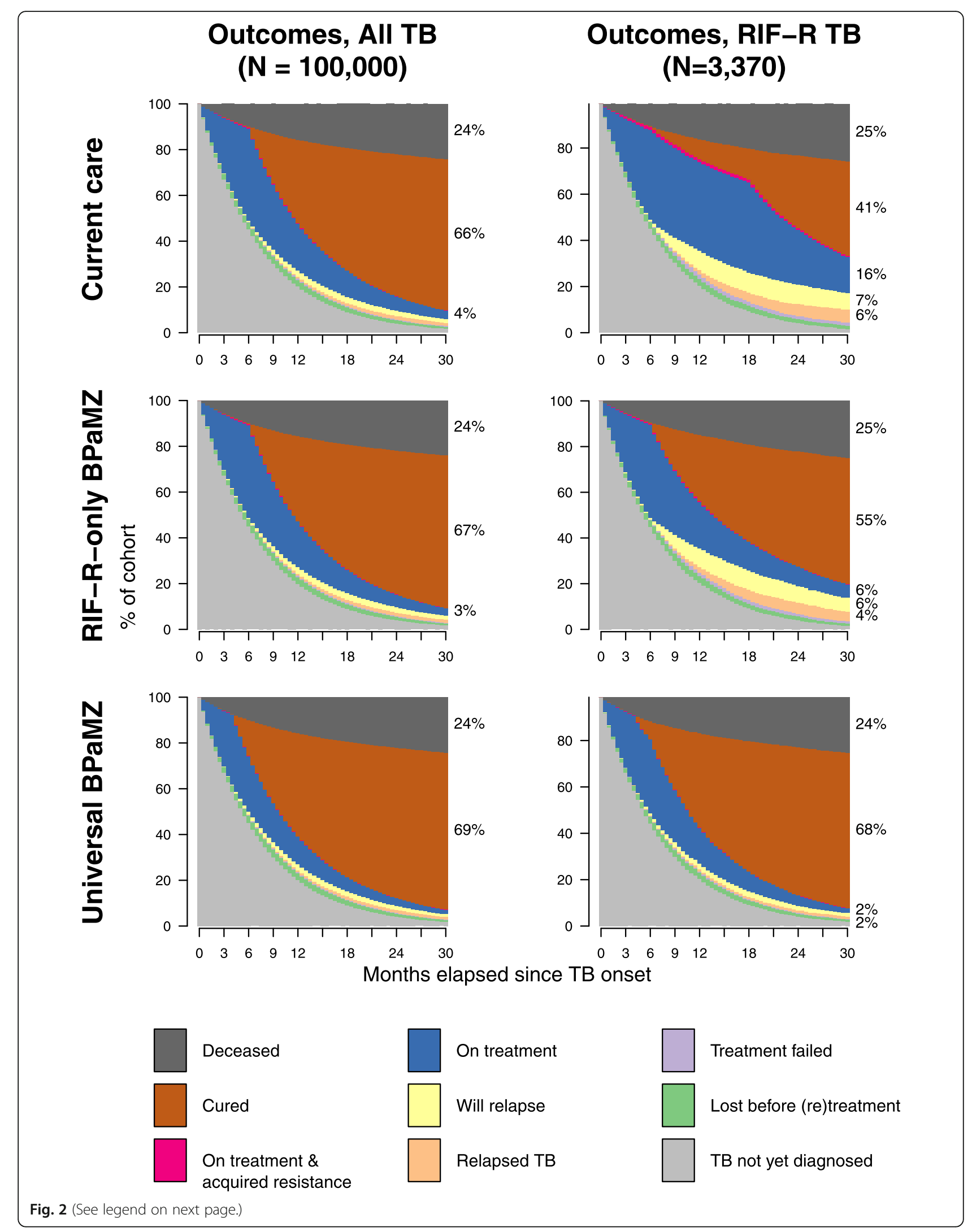


(See figure on previous page.)

Fig. 2 Simulated impact of BPaMZ regimen on status of South African TB cohort and RR sub-cohort over time. Scenarios modeled are: (a) standard of care baseline (including conventional DR-TB regimens for those found to have RR-TB, and HRZE for all others; top row), (b) introduction of the novel regimen for patients known to have RR-TB (middle row), and (c) introduction of the novel regimen for all patients (with duration dependent on the DST result if rifampin DST is performed; bottom row). The percentages along the right edge of each panel show the fraction of the cohort in each state 30 months after onset of TB (with fractions $<2 \%$ not labeled)

Current Care and $67.1 \pm 0.9 \%$ when BPaMZ was used for recognized RR-TB.

Using BPaMZ universally provided additional benefit, much of which also accrued to patients with RR-TB. The universal indication facilitated more effective treatment for the $48 \%$ of TB with RR that went undetected (due to non-bacteriologic TB diagnoses, incomplete Xpert coverage, or imperfect Xpert sensitivity). It thus further increased RR-TB cure after the first round of treatment, to $66.7 \pm 0.6 \%$ of all individuals with RR-TB and $88.8 \pm 0.6 \%$ of RR-TB patients treated with any TB treatment.

For individuals with RS-TB, treatment with Current Care achieved cure for $66.2 \pm 0.2 \%$ of all individuals with RS-TB and $88.4 \pm 0.1 \%$ of RS-TB patients treated. Universal use of $\mathrm{BPaMZ}$ increased these probabilities of RSTB cure by only $1 \%$, but it maintained high cure rates $67.0 \pm 0.2 \%$ of all individuals with RS-TB, or $89.5 \pm 0.1 \%$ of RS-TB patients treated - with the advantage of a shorter 4-month treatment duration.

Considering all individuals with $\mathrm{TB}$ and up to four rounds of treatment, the proportion ultimately cured of TB increased slightly from $77.8 \pm 0.1 \%$ with Current Care to $78.9 \pm 0.1 \%$ with Universal BPaMZ.

\section{Impact of $\mathrm{BPaMZ}$ regimen on treatment duration and medication use}

Comparing Universal BPaMZ to Current Care, the average cumulative treatment time fell from 5.4 months to 3.5 months per individual in the cohort (Additional file 1: Table S7). These totals include those never treated, those lost to follow up after partial treatment courses, and those requiring retreatments, among both RS- and RR-TB.

Per 1000 individuals with TB followed for up to four rounds of diagnosis and attempted treatment, a switch from Current Care to RR-only use of BPaMZ eliminated total $27 \pm 1$ months of treatment (with multidrug-resistant $\mathrm{TB}$ regimens) and replaced an additional $14 \pm 1$ months of conventional multidrug-resistant $\mathrm{TB}$ treatment with the same number of months of the BPaMZ regimen. Using BPaMZ universally, as opposed to only for RR-TB, further eliminated $170 \pm 1$ months of treatment (with the additional eliminated treatment months being months of the HRZE regimen) and replaced an additional $333 \pm 1$ months of HRZE with the same number of months of BPaMZ.

\section{Expected impact on transmission potential and drug resistance}

Compared to Current Care, Universal BPaMZ reduced the total time with active (and potentially-infectious) TB by $6.4 \pm 0.6$ months per person (a $41 \pm 3 \%$ reduction) among those who had RR-TB at the start of the model, and by $0.33 \pm 0.05$ months per case (a $3.5 \pm 0.6 \%$ reduction) in the overall TB cohort.

The use of BPaMZ resulted in an increase in bedaquiline and pretomanid resistance, and also had potential to increase moxifloxacin resistance when used as a universal regimen (Fig. 3). However, the amount of new drug resistance created could be small compared to the reduction in $\mathrm{RR}-\mathrm{TB}$, when measured in terms of potentially infectious person-time within a single TB cohort. Under our initial assumption of a relatively low risk of drug resistance acquisition during treatment of initially-pan-susceptible TB with BPaMZ, the switch from Current Care to Universal BPaMZ eliminated $14 \pm 1$ months of active RR-TB, while adding a combined $4 \pm 1$ months of active moxifloxacinresistant, bedaquiline-resistant, and/or pretomanid-resistant TB. In the sensitivity analysis with approximately fivefold higher estimated risks of resistance acquisition during BPaMZ treatment, the aggregate increase in time with moxifloxacin-resistant, bedaquiline-resistant, and/or pretomanid-resistant TB exceeded the reduction in time with RR-TB by a factor of two: $32 \pm 1$ months of active MXR-, bedaquiline-, or pretomanid-resistant TB added, in exchange for approximately the same $14 \pm 1$ months of RR-TB averted (Fig. 3). Finally, under the assumption of a nonzero (2\%) initial prevalence of bedaquiline resistance within the cohort (and low risk of resistance acquisition when initially pan-susceptible), overall bedaquiline-resistance transmission increased. However, because cure rates remained relatively high at $80.6 \pm 0.1 \%$ for bedaquiline-resistant TB (including polydrug resistant $\mathrm{TB}$ ), Universal BPaMZ caused relatively small increases in the expected transmission of bedaquiline-, moxifloxacin-, and pretomanid-resistant TB relative to expected transmission of bedaquiline-resistant and moxifloxacin-resistant TB under current care (Additional file 1: Figure. S1).

\section{Dependence of BPaMZ impact on local RR-TB epidemiology and detection}

In the South African setting modeled, the combined proportion of new and retreatment $T B$ cases with $R R$ was 


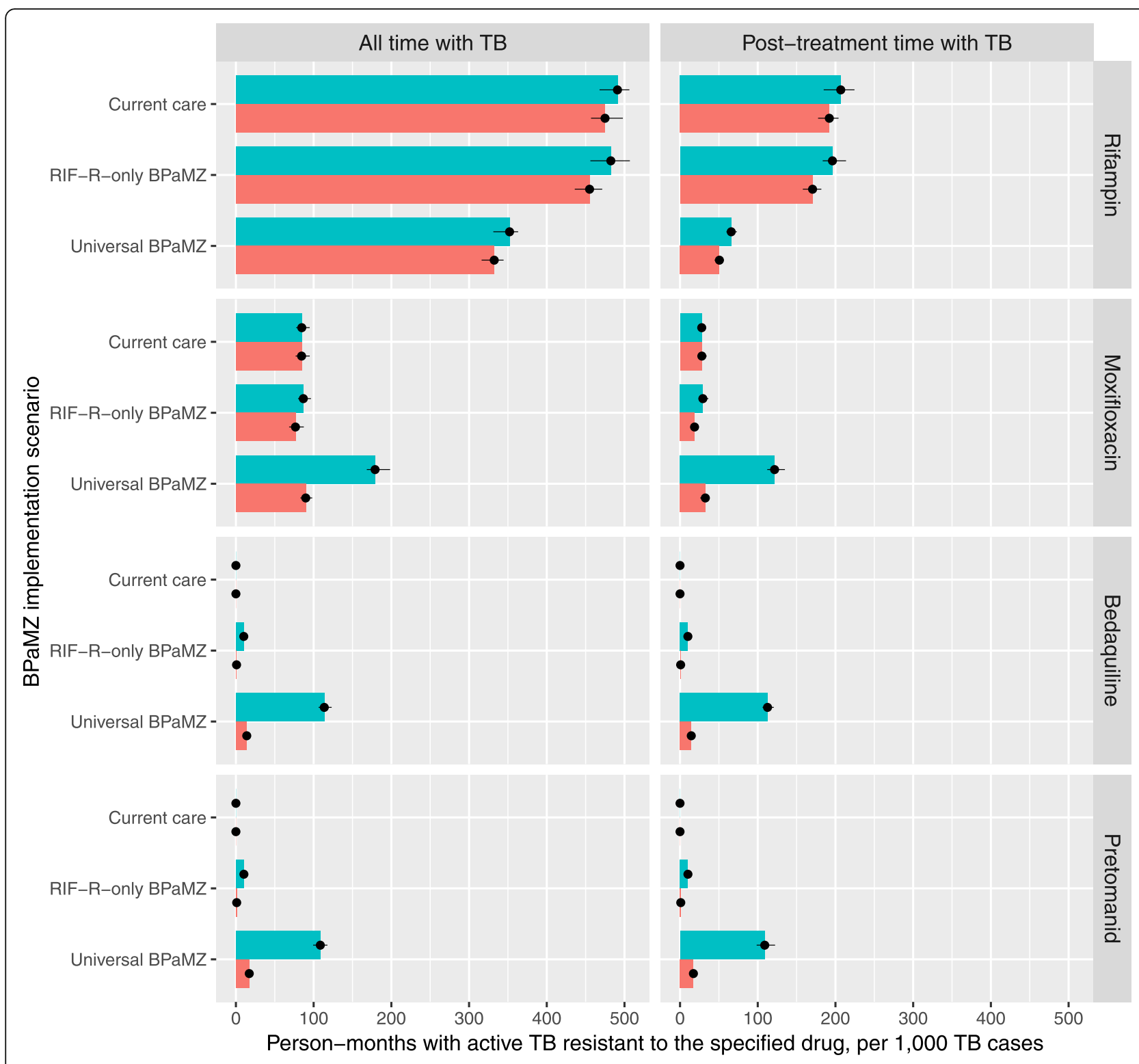

Risk of acquiring resistance: $\$$ Low $\$$ High

Fig. 3 Impact of BPaMZ regimen use and barrier to resistance on potentially infectious person-time. Total time with drug-resistant TB (left column) includes the time prior to the first treatment within the model, while the right column shows time with active drug-resistant TB after an individual has begun treatment at least once within the model - that is, time and potential transmission that better treatment might have prevented. For comparison, in the Current Care scenario, total time with TB (with or without drug resistance) was 8900 person-months overall and 1400 person-months after a treatment attempt, per 100 TB cases. Acquired resistance risk parameters increase risks of moxifloxacin, bedaquiline, and pretomanid resistance during $\mathrm{BPaMZ}$, and also of isoniazid resistance during HRZE

below the global average, and rifampin DST coverage was high. Varying these conditions had important effects on the expected impact of BPaMZ, and on the advantages of Universal versus RR-only BPaMZ use (Fig. 4).

First, increasing the prevalence of RR from 3.8\% to near $10.5 \%$, while maintaining high Xpert coverage, maintained the expectation of a high cure rate $(89.5 \pm$ $0.1 \%$ ) for Universal BPaMZ (Fig. 4). At the same time, this higher RR prevalence (combined with low DR-TB treatment success rates under Current Care) increased the extent to which Universal BPaMZ could improve upon the status quo (a $4.1 \pm 0.1 \%$ increase in overall cure rate versus Current Care, compared to a $2.2 \pm 0.1 \%$ increase with lower RR prevalence). Higher RR prevalence also increased the fraction of BPaMZ's total impact that could be achieved through use of BPaMZ for RR-TB 


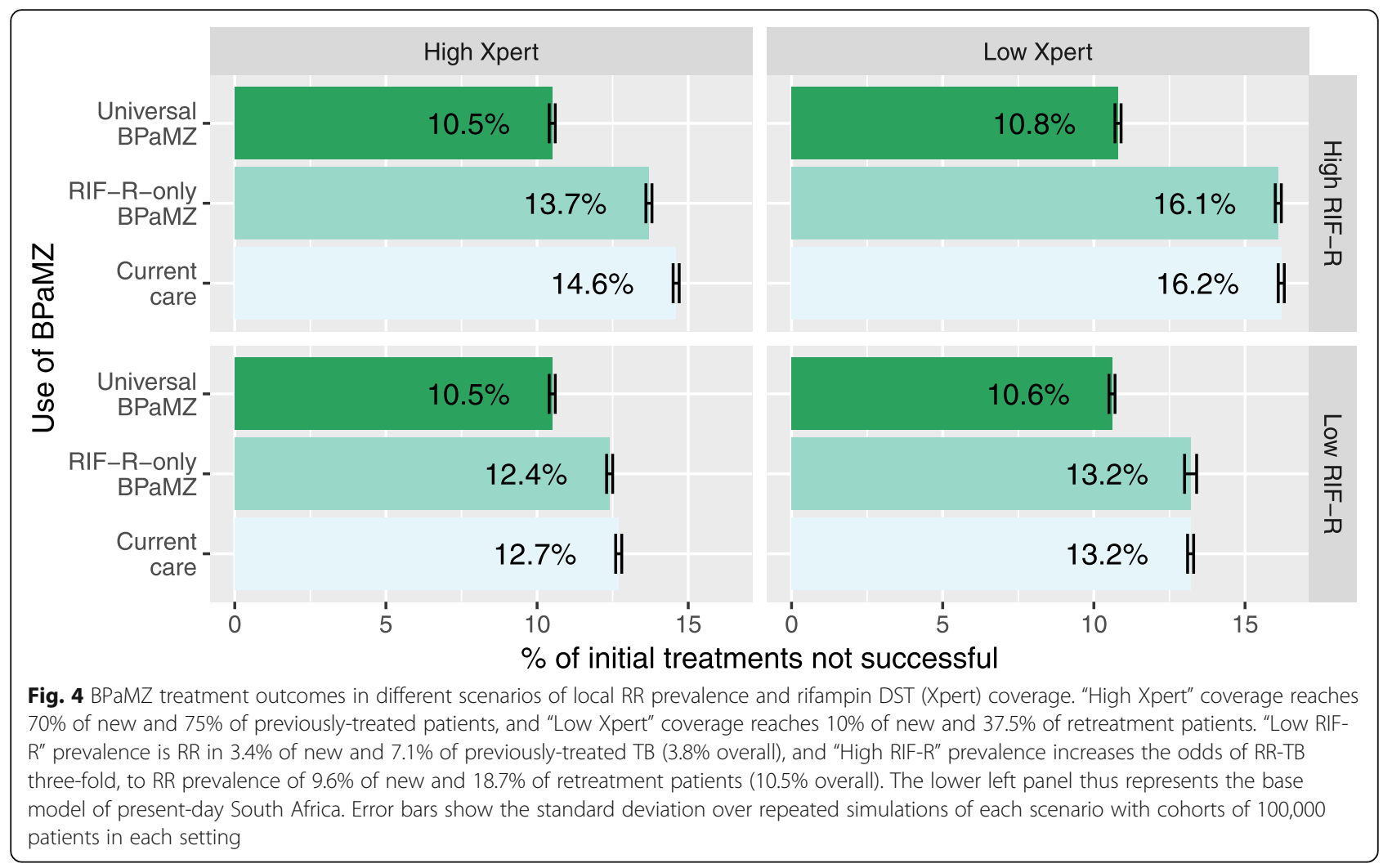

alone, although Universal BPaMZ use still accounted for most (approximately 85\%) of BPaMZ's potential to increase cure.

If Xpert coverage fell (reducing rifampin DST availability from 70 to $10 \%$ of new cases and from 75 to $37.5 \%$ of retreatment cases), then the impact of RR-only $\mathrm{BPaMZ}$ use on overall TB cure rates became negligible (remaining at $86.8 \pm 0.2 \%$ cure with low RR prevalence, and $85.9 \pm 0.1 \%$ with high RR prevalence, Fig. 4). Meanwhile, the benefits of Universal BPaMZ over Current Care grew, because the universal regimen benefitted TB patients with undetected RR. The benefits of Universal BPaMZ over Current Care were maximized in a setting where Xpert coverage was low and $R R$ prevalence simultaneously high (where it increased cure by $5 \%$ from $83.9 \pm 0.1 \%$ to $89.2 \pm 0.1 \%$, versus a $2 \%$ increase in the base model), even though such a scenario resulted in slightly more patients having poor BPaMZ treatment outcomes than in other settings $(10.8 \pm 0.1 \%$ of patients, compared to $10.5 \pm 0.1 \%$ in the base model, as a result of more patients with moxifloxacin-resistant and/or pyrazinamide-resistant TB receiving only 4 months of BPaMZ).

\section{Additional scenario and sensitivity analyses}

We repeated the analysis with three-fold higher odds of moxifloxacin and pyrazinamide resistance - a change which increased the prevalence of moxifloxacin resistance and pyrazinamide resistance in the cohort to 4 and $1 \%$, respectively, among RIF-S TB, and to 70 and $25 \%$ among RR-TB, leading to resistance resembling the former Soviet Union region in the model scenario with high RR prevalence as well. This change reduced BPaMZ cure rates by $<0.5 \%$ in the reference model, and by 0.5 to $1 \%$ in settings with high RR prevalence and/or low Xpert coverage (Additional file 1: Figure. S3).

With higher estimated probabilities of acquiring moxifloxacin, bedaquiline, or pretomanid resistance during BPaMZ treatment, initial cure rates remained unchanged, but among those not cured by initial treatment, the prevalence of drug resistance increased, leading to worse outcomes during retreatment. Thus, the proportion of all individuals with $\mathrm{TB}$ who were cured within two rounds of attempted treatment with Universal BPaMZ fell slightly from $77.1 \pm 0.1 \%$ to $76.9 \pm$ $0.1 \%$; among patients with RR-TB, this proportion fell from $76.6 \pm 0.7 \%$ to $73.2 \pm 0.8 \%$. Considering only those who were retreated after not being cured by initial treatment, higher estimates of acquired resistance reduced the proportion cured from $88.9 \pm 0.5 \%$ to $85.8 \pm 0.3 \%$ overall, and from $86.4 \pm 1.3 \%$ to $65.6 \pm$ $2.4 \%$ for initial RR-TB (where pyrazinamide resistance or moxifloxacin resistance were a contributor in most initial failures to cure). 
Lowering BPaMZ efficacy (to 91\% non-relapsing cure after 4 months if pan-susceptible - a value potentially still within clinical trial noninferiority margins) lowered the expected Universal BPaMZ cure rates in South Africa from $89.5 \pm 0.1 \%$ to $87.3 \pm 0.1 \%$ and prevented BPaMZ from improving treatment outcomes over Current Care in most settings (Additional file 1: Figure. S4).

Finally, improving the standard of care for RR-TB prior to BPaMZ introduction reduced the benefits of BPaMZ among patient with RR-TB, but maintained some benefit: among RR-TB patients who received any treatment, the percentage cured with RR-only implementation of BPaMZ rose by only 3\% (from 64 to $67 \%$ ) relative to the improved RR-TB standard of care, compared to the $7 \%$ increase with BPaMZ relative to Current Care (from 60 to 67\%) in our original analysis. Because patients receiving the drug-resistant-TB-specific regimen accounted for a small fraction of total poor outcomes, BPaMZ's impact on overall cure rates was minimally affected (Additional file 1: Figure. S5).

\section{Discussion}

If the outstanding performance of the BPaMZ regimen in preliminary studies is confirmed in larger-scale trials, it could offer an important advance by shortening treatment durations using a single drug combination for nearly all patients with TB. While patients with RR-TB would derive the greatest benefits, using such a regimen universally (for RS- and well as RR-TB) could offer important additional benefits for both groups of patients: for patients with RS-TB, it would shorten the treatment duration, while for patients with RR-TB that might be missed by DST practices, it could substantially increase cure. Modeling a South African TB cohort, we have estimated that implementing the BPaMZ regimen universally (with stratification of treatment duration based on rifampin susceptibility) could simultaneously increase the percentage of patients with RR-TB who are cured from $60 \%$ to nearly $90 \%$, maintain nearly $90 \%$ cure among patients with RS-TB, reduce treatment duration by 2 months or more per patient, and reduce infectious person-time by $3 \%$ (RS-TB) to $50 \%$ (RR-TB). The potential impact of an effective universal regimen is even greater in settings of higher RR prevalence or lower DST coverage.

As BPaMZ or similar regimens become available, TB programs and health systems will need to choose whether to implement such regimens, and whether to restrict their use to patients with RR-TB. Our model shows that the impact of $\mathrm{BPaMZ}$ as an RR-only $\mathrm{TB}$ treatment regimen depends heavily on the extent to which RR-TB is consistently detected. For settings with moderate or high $\mathrm{RR}$ prevalence that cannot achieve extremely high rates of $R R$, regimen universalization is expected to increase the impact on cure several-fold. Additional advantages of universal implementation would include shorter treatment duration and streamlined care delivery, with benefits for patients and providers. Affordability evaluations will need to consider both the potentially higher costs of replacing low-cost HRZE with novel drugs, and the possible reductions in costs of health care delivery [28].

Acquired resistance and resistance transmission are a potential concern with use of any new drug combination. Our analysis suggests that, although use of a bedaquiline- and pretomanid- containing regimen is expected to create some resistance to these drugs and will require a resistance-management strategy, this is not a compelling reason to avoid universal use in the short term as long as current efficacy estimates for BPaMZ are borne out. For example, even if probabilities of acquiring bedaquiline or pretomanid resistance turn out to be relatively high, initial increases in bedaquiline-resistant or pretomanid-resistant TB transmission could be counterbalanced by reduced RR-TB transmission; in addition, most acquired bedaquiline- and pretomanid resistance would occur in RS-TB strains for which other treatment options would still exist as long as the bedaquiline resistance were identified [29]. Our conclusions resemble those of a previous analysis which favored use of bedaquiline for all multidrug-resistant $\mathrm{TB}$ rather than only extensively-drug-resistant TB despite the increase in acquired bedaquiline resistance [30]. However, over several years and several cycles of transmission, compounding novel-drug resistance could pose a barrier to universal use of this novel regimen, with a moderately low barrier to resistance potentially resulting in novel-drug resistance in up to $5 \%$ of $\mathrm{TB}$ cases after 5 years and more than $10 \%$ of TB cases after 50 years under pessimistic assumptions and drug resistance transmission [31]. Our results therefore highlight the need to better quantify risks of spontaneously-occurring [26] and acquired resistance to these drugs, and to take appropriate actions to detect and contain resistance. Initial steps to preserve and maximize regimen usefulness include drug-resistance surveillance at the population level, and patientlevel identification of risk factors such as previous treatment with BPaMZ (for example, for RR-TB in our model, BPaMZ cure rates were 3 to $30 \%$ lower in retreatment than initial treatment, depending on the estimated risks of BPaMZ resistance acquisition) or treatment with other drugs with potential cross-resistance (e.g., delamanid or clofazimine) [27, 32]. Acquisition of bedaquiline or pretomanid resistance would not mean we must avoid using these drugs, but it would indicate an urgent need to develop strong drug resistance surveillance systems and rapid DST. 
Our findings should be interpreted in light of certain limitations. First, because no human relapse data yet exist for BPaMZ, projected cure rates must be extrapolated from animal data, early-phase clinical studies, and clinical experience with other TB drugs. It will be important to glean better estimates and revise projections based on phase $2 c / 3$ study results - and, if the regimen succeeds, to conduct post-approval studies of outcomes in rarer but important patient situations such as moxifloxacin-resistant TB. A second important limitation is the lack of explicit representation of TB transmission in this model, although we do look at potentially infectious person-time as a first order approximation. This approach allowed us to model relationships between assumptions and outcomes (including infectious person-time) more transparently, at the expense of neglecting effects on population-level transmission over time, and allowed us to model a large number of different patient characteristics. We considered TB-related outcomes and time on treatment but not drug side effects or safety monitoring, which will differ between regimens and also should be taken into account in clinical and cost-effectiveness evaluations. Finally, for the current analysis we limited our consideration of DST to the use of RR in selecting eligible patients or optimal treatment durations, but DST for fluoroquinolones or pyrazinamide could also have a useful role alongside such a regimen.

\section{Conclusions}

Novel drugs are reshaping drug-resistant TB treatment and could soon transform the broader TB treatment landscape if ongoing clinical trial evaluations identify regimens that improve upon current first-line treatment. Our modeling results show that if BPaMZ or a similar regimen is effective in trials of long-term cure, it could offer important benefits for TB treatment success rates, $\mathrm{TB}$ treatment duration, and streamlined TB regimen selection. If such a regimen were used for RR-TB only, the benefits for those patients who received it could be large compared to continued use of conventional MDR-TB regimens. On a population level, as long as emerging resistance to new drugs is appropriately identified and managed, our analysis suggests that universal use of such a regimen would amplify its benefits for patients both with and without rifampin resistance and across a range of drug-resistance epidemiology.

\section{Additional file}

Additional file 1: Supplemental Material. Contains supplementary methods including a complete description of the model, and supplementary results tables and figures. (DOCX $234 \mathrm{~kb}$ )

\section{Abbreviations}

BPaMZ: Bedaquiline, pretomanid, moxifloxacin, and pyrazinamide; DST: Drug susceptibility testing; HRZE: The current first line regimen of isoniazid, rifampin, pyrazinamide, and ethambutol; RR: Rifampin resistant; RS: Rifampin susceptible; TB: Tuberculosis

\section{Acknowledgements}

Not applicable.

\section{Authors' contributions}

EAK, SM, SCS, CMD, and DWD contributed to conceptualization of the study and critical revision of the manuscript. SM contributed clinical trial data. EAK curated data, designed and coded the model, performed computational analysis and interpreted results, developed figures and tables, and prepared the first draft of the manuscript with supervision from DWD. All authors have read and approved the final version of the manuscript

\section{Funding}

This work was supported by a US National Institutes of Health career development award K08Al127908 to EAK. The funding source had no role in the design of the study, the collection, analysis, or interpretation of the data, or the writing of the manuscript.

\section{Availability of data and materials}

The datasets used and analysed during the current study are. available from the corresponding author on reasonable request.

\section{Ethics approval and consent to participate}

Not applicable.

\section{Consent for publication}

Not applicable.

\section{Competing interests}

SCS is a current employee and SM is a former employee of TB Alliance, the not-for-profit product development partnership leading development of the BPaMZ regimen.

\section{Author details}

${ }^{1}$ Division of Infectious Diseases and Center for Tuberculosis Research, Johns Hopkins University School of Medicine, CRB2 Room 106, 1550 Orleans St, Baltimore, MD 21287, USA. ${ }^{2}$ Global Alliance for TB Drug Development, New York, NY, USA. ${ }^{3}$ International AIDS Vaccine Initiative, New York, NY, USA. ${ }^{4}$ Division of Tropical Medicine, Center of Infectious Disease, Heidelberg University, Heidelberg, Germany. ${ }^{5}$ Tuberculosis Programme, FIND, Geneva, Switzerland. ${ }^{6}$ Department of Epidemiology, Johns Hopkins Bloomberg School of Public Health, Baltimore, MD, USA.

Received: 9 July 2019 Accepted: 30 August 2019

Published online: 09 September 2019

\section{References}

1. World Health Organization. Global tuberculosis report 2018. Geneva: World Health Organization; 2018.

2. Romanowski K, Balshaw RF, Benedetti A, Campbell JR, Menzies D, Ahmad Khan F, et al. Predicting tuberculosis relapse in patients treated with the standard 6-month regimen: an individual patient data meta-analysis. Thorax. 2019;74:291-7.

3. Lienhardt C, Nahid P, Rich ML, Bansbach C, Kendall EA, Churchyard G, et al. Target regimen profiles for treatment of tuberculosis: a WHO document. Eur Respir J. 2017:49:1602352.

4. Dawson R, Harris K, Conradie A, Burger D, Murray S, Mendel C, et al. Efficacy of Bedaquiline, Pretomanid, Moxifloxacin and PZA (BPaMZ) Against DS- and MDR-TB. In: CROI 2017 Abstract eBook [Internet]; 2017 Feb 13-16; Seattle: CROI Foundation/International Antiviral Society-USA; 2017 [cited 2019 Sept 5]; p. 316. Available from: https://www.croiconference.org/sites/default/files/ uploads/croi2017-abstract-eBook.pdf.

5. Li S-Y, Tasneen R, Tyagi S, Soni H, Converse PJ, Mdluli K, et al. Bactericidal and sterilizing activity of a novel regimen with Bedaquiline, Pretomanid, moxifloxacin, and pyrazinamide in a murine model of tuberculosis. Antimicrob Agents Chemother. 2017;61:e00913-7. 
6. Clinicaltrials.gov. Trial to Evaluate the Efficacy, Safety and Tolerability of BPaMZ in Drug-Sensitive (DS-TB) Adult Patients and Drug-Resistant(DR-TB) Adult Patients [Internet]. US National Library of Medicine; 4 Sept 2019 [cited 5 Sept 2019]. Available from: https://clinicaltrials.gov/ct2/show/NCT0333 8621.

7. Zignol M, Dean AS, Alikhanova N, Andres S, Cabibbe AM, Cirillo DM, et al. Population-based resistance of mycobacterium tuberculosis isolates to pyrazinamide and fluoroquinolones: results from a multicountry surveillance project. Lancet Infect Dis. 2016;16:1185-92.

8. Centre for Tuberculosis. South African Tuberculosis Drug Resistance Survey 2012-14 [Internet]. Johannesburg: National Institute for Communicable Diseases Division of the National Health Laboratory Service; 2016 [cited 2019 Sept 5]. Available from: http://www.nicd.ac.za/assets/files/K-12750\%2 ONICD\%20National\%20Survey\%20Report_Dev_V11-LR.pdf.

9. World Health Organization. WHO TB burden estimates [Internet]. Geneva; 2019 [cited 2019 Sept 5]. Available from: https://www.who.int/tb/country/ data/download/en/.

10. Ministry of Health and Family Welfare, World Health Organization India, DOTS, National Health Mission, USAID. Report of the first National Anti TB drug resistance survey, India 2014-2016.

11. Subbaraman R, Nathavitharana RR, Satyanarayana S, Pai M, Thomas BE, Chadha VK, et al. The tuberculosis Cascade of Care in India's public sector: a systematic review and meta-analysis. PLoS Med. 2016;13:e1002149.

12. Kruk ME, Schwalbe NR, Aguiar CA. Timing of default from tuberculosis treatment: a systematic review. Tropical Med Int Health. 2008;13:703-12.

13. Tiemersma EW, van der Werf MJ, Borgdorff MW, Williams BG, Nagelkerke NJD. Natural history of tuberculosis: duration and fatality of untreated pulmonary tuberculosis in HIV negative patients: a systematic review. PLoS One. 2011;6:e17601.

14. Merle CS, Fielding K, Sow OB, Gninafon M, Lo MB, Mthiyane T, et al. A fourmonth gatifloxacin-containing regimen for treating tuberculosis. N Engl J Med. 2014;371:1588-98.

15. Gillespie SH, Crook AM, McHugh TD, Mendel CM, Meredith SK, Murray SR, et al. Four-month moxifloxacin-based regimens for drug-sensitive tuberculosis. N Engl J Med. 2014;371:1577-87.

16. Jindani A, Harrison TS, Nunn AJ, Phillips PPJ, Churchyard GJ, Charalambous $\mathrm{S}$, et al. High-dose rifapentine with moxifloxacin for pulmonary tuberculosis. N Engl J Med. 2014;371:1599-608.

17. Ahmad N, Ahuja SD, Akkerman OW, Alffenaar J-WC, Anderson LF, Baghaei P, et al. Treatment correlates of successful outcomes in pulmonary multidrugresistant tuberculosis: an individual patient data meta-analysis. Lancet. 2018; 392:821-34.

18. Menzies D, Benedetti A, Paydar A, Martin I, Royce S, Pai M, et al. Effect of duration and intermittency of rifampin on tuberculosis treatment outcomes: a systematic review and meta-analysis. PLoS Med. 2009;6:e1000146.

19. Cegielski JP, Kurbatova E, van der Walt M, Brand J, Ershova J, Tupasi T, et al. Multidrug-resistant tuberculosis treatment outcomes in relation to treatment and initial versus acquired second-line drug resistance. Clin Infect Dis. 2016;62:418-30.

20. Dawson R, Diacon AH, Everitt D, van Niekerk C, Donald PR, Burger DA, et al. Efficiency and safety of the combination of moxifloxacin, pretomanid (PA824), and pyrazinamide during the first 8 weeks of antituberculosis treatment: a phase $2 \mathrm{~b}$, open-label, partly randomised trial in patients with drug-susceptible or drug-resistant pulmonary tuberculosis. Lancet. 2015;385: 1738-47.

21. Wallis RS, Peppard T, Hermann D. Month 2 culture status and treatment duration as predictors of recurrence in pulmonary tuberculosis: model validation and update. PLoS One. 2015:10:e0125403.

22. Ahuja SD, Ashkin D, Avendano M, Banerjee R, Bauer M, Bayona JN, et al. Multidrug resistant pulmonary tuberculosis treatment regimens and patient outcomes: an individual patient data meta-analysis of 9,153 patients. PLoS Med. 2012;9:e1001300.

23. Organization WH. WHO treatment guidelines for drug-resistant tuberculosis: 2016 update. 2016

24. Nunn AJ, Phillips PPJ, Meredith SK, Chiang C-Y, Conradie F, Dalai D, et al. A trial of a shorter regimen for rifampin-resistant tuberculosis. N Engl J Med. 2019;380:1201-13.

25. Ndjeka N, Schnippel K, Master I, Meintjes G, Maartens G, Romero R, et al. High treatment success rate for multidrug-resistant and extensively drugresistant tuberculosis using a bedaquiline-containing treatment regimen. Eur Respir J. 2018;52:1801528.
26. Villellas C, Coeck N, Meehan CJ, Lounis N, de Jong B, Rigouts L, et al. Unexpected high prevalence of resistance-associated Rv0678 variants in MDR-TB patients without documented prior use of clofazimine or bedaquiline. J Antimicrob Chemother. 2016;72:dkw502.

27. Hartkoorn RC, Uplekar S, Cole ST. Cross-resistance between clofazimine and bedaquiline through upregulation of MmpL5 in mycobacterium tuberculosis. Antimicrob Agents Chemother. 2014;58:2979-81.

28. Kendall EA, Brigden G, Lienhardt C, Dowdy DW. Would pan-tuberculosis treatment regimens be cost-effective? Lancet Respir Med. 2018;6(7):486-8.

29. Kunkel A, Furin J, Cohen T. Population implications of the use of bedaquiline in people with extensively drug-resistant tuberculosis: are fears of resistance justified? Lancet Infect Dis. 2017;17(12):e429-33.

30. Kunkel A, Cobelens FG, Cohen T. Tradeoffs in introduction policies for the anti-tuberculosis drug Bedaquiline: a model-based analysis. PLoS Med. 2016; 13:e1002142.

31. Shrestha S, Knight GM, Fofana M, Cohen T, White RG, Cobelens F, et al. Drivers and trajectories of resistance to new first-line drug regimens for tuberculosis. Open Forum Infect Dis. 2014;1:ofu073.

32. Rifat D, Li S-Y, loerger T, Lanoix J-P, Lee J, Bashiri G, et al. Mutations in Rv2983 as a novel determinant of resistance to nitroimidazole drugs in mycobacterium tuberculosis. bioRxiv. 2018;1:457754.

\section{Publisher's Note}

Springer Nature remains neutral with regard to jurisdictional claims in published maps and institutional affiliations.

\section{Ready to submit your research? Choose BMC and benefit from:}

- fast, convenient online submission

- thorough peer review by experienced researchers in your field

- rapid publication on acceptance

- support for research data, including large and complex data types

- gold Open Access which fosters wider collaboration and increased citations

- maximum visibility for your research: over $100 \mathrm{M}$ website views per year

At $\mathrm{BMC}$, research is always in progress.

Learn more biomedcentral.com/submissions 